\title{
MODERNES RECHT \\ UND TRADITIONELLE GESELLSCHAFTEN
}

\author{
FranZ von BENDA-BeCKMANN
}

In entwickelten Gesellschaften, wie in Holland oder in der Schweiz, haben Juristen wie Nichtjuristen meist das Gefühl, in einer modernen Gesellschaft mit einem modernen Recht zu leben. Dies Gefühl wird vor allem dann aktualisiert, wenn man seine eigene Gesellschaft und ihr Recht mit den traditionellen Gesellschaften in den Ländern der Dritten Welt und ihren meist ungeschriebenen Stammes- oder Gewohnheitsrechten vergleicht. Was man sich dabei unter ,Modernität“" vorstellt, wird oft unterschiedlich sein. Der eine denkt an Autos, Farbfernsehen, an Elektrizität, Kunstdünger, Industrialisierung; der andere drückt sich abstrakter aus und spricht von sozialer Differenzierung, funktioneller Spezialisierung und zunehmender sozialer Komplexität als den wesentlichen Kriterien von Modernität. ${ }^{1}$ Länder wie Holland erfüllen diese Bedingungen weitgehend. Anders jedoch die traditionellen Gesellschaften in der Dritten Welt. Die gerade genannten technischen Errungenschaften findet man dort kaum. Auch weiß man um den sehr geringen Grad an sozialer Differenzierung in diesen Gesellschaften: Das Individuum ist dort in ein Netz von vielschichtigen sozialen Beziehungen eingebettet, die kaum voneinander zu trennen sind, wie Verwandtschaftsbeziehungen, wirtschaftliche, religiöse und politische Beziehungen. Bekannt ist ebenfalls der geringe Grad an funktioneller Spezialisierung: Familien-, Clan- oder Dorfälteste treten gleichzeitig als Oberhäupter von Verwandtschaftsgruppen, von wirtschaftlichen oder politischen Einheiten auf. Sie sind die höchsten politischen Organe ihrer Gesellschaften, und in ihren Händen liegen all die Funktionen, die wir als legislative, judikative und exekutive unterscheiden würden.

Dieser Zustand gehört natürlich schon weitgehend der Vergangenheit an. Fast alle traditionellen Gesellschaften sind seit langer Zeit Teile von Kolonialgebieten und jetzt von unabhängigen Staaten. Die Kolonialmächte bauten ein neues Verwaltungssystem auf, führten ihr $R \epsilon$ cht und eigene Gerichte ein und beschränkten den Geltungsumfang der lokalen ungeschriebenen Rechte. ${ }^{2}$ Westliche kulturelle und religiöse Wertvorstellungen wurden der Bevölkerung nahegebracht, oft aufgedrängt. Weiter wurden die traditionellen Wirtschaftssysteme in einem immer noch fortschreitenden Prozeß durch das internationale kapitalistische System beeinflußt und zum Teil desselben gemacht. Dies ist, in wenigen Worten, der Hintergrund, vor dem ich auf das Problem der Rechtsentwicklung in tradionellen Gesellschaften eingehen möchte. Bei dem Wort Rechtsentwicklung denke ich hier an zwei unterschiedliche Prozesse. Einmal geht es um die Anpassung der traditionellen Gewohnheitsrechte an die veränderten sozialen, kulturellen, wirtschaftlichen und politischen Verhältnisse; und damit zugleich um das Verhältnis zwischen Rechtsentwicklung einerseits und sozialer und wirtschaftlicher Entwicklung andererseits. Zum anderen geht es um Rechtsentwicklung im Sinne einer bewußt geplanten Veränderung bestehender Normen mit dem Ziele, mit Hilfe des

\footnotetext{
Dieser Beitrag ist die ijberarbeitete Fassung einer Gastvorlesung, die der Verfasser am 9. 7. 1979 auf Einladung der Sozialwissenschaftlichen Fakultät I der Universität Zürich gehalten hat.

1 Vergl. Bryde 1977: 121. Apter 1965: $43 \mathrm{ff}$.

2 Einen guten Ưberblick über die kolonialen Rechtssysteme gibt Hooker 1975. Für Afrika s. Elias 1962, Allott 1970. Für eine detaillierte Beschreibung eines einzelnen ehemaligen englischen Kolonialgebiets (Nyasaland) s. von Benda-Beckmann 1970.
} 
Rechts bestimmte sozial- oder wirtschaftspolitische Wunschvorstellungen zu fördern oder durchzusetzen. ${ }^{3}$ Die Regierungen vieler Staaten der Dritten Welt gehen immer mehr dazu über, Recht, meist in der Form von Gesetzen, als Mittel für soziale und wirtschaftliche Veränderungen einzusetzen. Hiervon zeugen zahlreiche Gesetze auf dem Gebiet des Familienund Bodenrechts. Der Form, aber auch dem Inhalt nach, wird hier überwiegend mit ,,modernem" Recht gearbeitet; ,,modern" steht hier für das Recht der westlichen Industriegesellschaften. Eine moderne, gleich westliche, Rechtsordnung erscheint den meisten Regierungen wünschenswert, zumindest aber unvermeidlich, will man das eigene soziale und wirtschaftliche System ,,entwickeln" und ,,modernisieren“."4

Ich kann in meinem Vortrag nicht alle wichtigen Aspekte dieses Problemfeldes behandeln. Ich möchte auch von vornherein eingestehen, daßich hier keine allgemeingültigen Aussagen darüber machen kann, unter welchen Voraussetzungen dergleiche Rechtsänderungen wünschenswert und erfolgreich sein könnten. Ich will mich darauf beschränken, an Hand eines konkreten Beispieles etwas Klarheit in den Zusammenhang zwischen Recht und nicht-rechtlicher Entwicklung in traditionellen Gesellschaften zu bringen. Ich hoffe, mit meinem Vortrag deutlich machen zu können, daß das Verständnis dieses Zusammenhangs die Ausgangsbasis für alle weiteren Úberlegungen über Rechtsentwicklung sein muß und daß es den gegenwärtigen Diskussionen noch sehr an diesem Verständnis gebricht.

Zunächst will ich das Problemfeld mit einem kleinen vergleichenden Beitrag illustrieren. Es geht dabei um den Bereich der individuellen Verfügungsfreiheit über Vermögen. Die Art, in der unterschiedliche Rechtssysteme diese Freiheit festlegen, macht zugegebenermaßen nur einen kleinen Bereich des Zivilrechts aus; dieser Bereich ist jedoch von großer Bedeutung für soziale und wirtschaftliche Beziehungen. Zugleich ist die Verfügungsfreiheit ein wichtiger Indikator von Modernität, da sie einen bestimmten Grad sozialer Differenzierung ausdrückt: Hier zeigt sich, inwieweit Individuen hinsichtlich ihrer Vermögensrechte und -pflichten von anderen sozialen Beziehungen, z. B. Verwandtschaftsbeziehungen, frei sind. Dieser Punkt spielt auch eine große Rolle in den Diskussionen über die wirtschaftliche Entwicklung in den Ländern der Dritten Welt. Ein geringes Maß an individueller Verfügungsfreiheit oder, komplementär ausgedrückt, eine starke Beschränkung dieser Verfügungsfreiheit durch politische und Verwandtschaftsbeziehungen, wird meist als ein wichtiges Hindernis auf dem Wege zu einer modernen und entwickelten Gesellschaft betrachtet.

Meine Illustration, mit der ich die Problematik näher veranschaulichen möchte, besteht aus einem Streitfall, der in zwei verschiedenen Gesellschaften entschieden wurde.

Es war einmal ein reicher Händler. Er besaß drei Läden in einer mittelgroßen Stadt, außerdem noch einigen Grundbesitz in einem nahegelegenen Dorf. Als er starb, ließ er eine Witwe und zwei Töchter nebst drei Kindern seiner ersten, schon vor längerer Zeit verstorbenen Frau zurück. Die Witwe und ihre Töchter hatten nach seinem Tod das ganze Vermögen an sich genommen. Als die Kinder der ersten Frau von ihres Vaters Tode hörten, gingen sie zu ihrer Stiefmutter, um mit ihr und ihren Halbgeschwistern über die Verteilung des Nachlasses zu sprechen. Aber da bekamen sie sehr unangeneme Neuigkeiten zu hören. Der Vater, so

\footnotetext{
3 Diese beide Formen entsprechen in etwa den Formen vonlaw reform, die Merryman ,,following “ und ,leading la w reform" nennt (1977: 462). Merryman unterscheidet daneben noch eine dritte Form, , tinkering “; die Situation, in der man das bestehende System als solches akzeptiert und nur gelegentlich Veränderungen anbringt, um die Effizienz des Systems zu verbessern (1977: 462).

4 S. Könz 1969, Merryman 1977, Burg 1977, Bryde 1977. Für konkrete Beispiele s. z. B. die Erbsrechts- und Bodenrechtsgesetze in Malawi, von Benda-Beckmann 1970: 108 ff. und 119 ff. Uber die in Indonesien herrschenden rechtspolitischen Vorstellungen s. BPHN 1974, 1975.
} 
sagten die Witwe und ihre Töchter, habe nämlich fast gar nichts hinterlassen. Den einen Laden habe er schon vor vielen Jahren an seine Tochter und deren Mann verkauft; einen anderen Laden habe er vor drei Jahren seiner Frau, der Witwe, geschenkt; den dritten Laden schließlich und den Grundbesitz habe er vor seinem Tode seiner jüngsten Tochter geschenkt. Und sie ließen die notariellen Urkunden sehen, in denen diese Transaktionen beglaubigt waren. Das Vermögen, wovon die drei Kinder der ersten Frau einen Teil als Erbteil meinten beanspruchen zu können, seien deshalb gar kein Erbgut; schon vor des Vaters Tode seien diese Sachen Eigentum der Witwe und ihrer Töchter geworden.

Als den drei Kindern deutlich wurde, daß die Witwe und ihre Halbschwestern nicht bereit waren, ihnen einen Anteil abzugeben, zogen sie vor Gericht, um dort ihr Recht zu suchen. In ihrer Klage führten sie an, daß all die Vermögensgegenstände das Eigentum ihres Vaters gewesen seien und deshalb, nach seinem Tode, zu seinem Nachlaß gehörten. Alle Rechtsgeschäfte, die ihr Vater zu Lebzeiten mit seiner Frau und seinen Töchtern getätigt habe, seien ungültig, oder könnten zumindest für ungültig erklärt werden. Denn deutlich seien es Scheinhandlungen gewesen, um sie als rechtmäßige Erben auszuschálten. Die beklagte Witwe und ihre Töchter wiederholten vor Gericht, was sie schon zuvor gesagt hatten. Sie legten die notariellen Urkunden vor und behaupteten, die zur Diskussion stehenden Vermögenswerte seien schon vor dem Tod des Vaters ihr Eigentum geworden. Von Erbansprüchen ihrer Halbgeschwister könne darum keine Rede sein.

In den beiden Gesellschaften wurden nun folgende Urteile gefällt.

\section{Gesellschaft A:}

Das Gericht in Gesellschaft A wies die Klage ab. Es hielt die Ubertragung der Sachen durch Verkauf und Geschenk für rechtswirksam. Nach dem in ihrer Gesellschaft geltenden Recht, so führte das Gericht aus, hätten die klagenden Kinder kein Recht, um nach dem Tode des Händlers noch etwas von den verkauften und verschenkten Sachen zurückzuverlangen. Diese Sachen waren sein Eigentum gewesen. Solange er lebte, konnte er frei darüber verfügen. Zwar gehörten auch die klagenden Kinder zu des Vaters rechtmäßigen Erben, ihr Erbrecht konnte jedoch erst mit und nach seinem Tode entstehen. Als der Verstorbene sein Vermögen verschenkte und verkaufte, war ein Erbrecht der Kinder also noch nicht entstanden. Nach dem in Gesellschaft A geltenden Recht sei es nie der Fall gewesen, daß jemand bei dergleichen Rechtshandlungen die Zustimmung seiner zukünftigen Erben habe einholen müssen. Die Vermögensgegenstände seien in diesem Fall also schon vor dem Tod in das Eigentum der Beklagten übergegangen und fielen damit nicht in den Nachlaß. Die Klage war darum abzuweisen.

\section{Gesellschaft B:}

Das Gericht in der Gesellschaft B gab der Klage weitgehend statt. Die Kläger bekamen (die Details lasse ich hier außer acht) fast ebenso große Anteile an dem Vermögen des Vaters zugesprochen wie die Witwe und ihre beiden Töchter. Mit der folgenden Begründung: Zwar entstehe ein Erbrecht für die Kinder erst mit dem Tode des Vaters; auch sei dessen Verfügungsfreiheit zu Lebzeiten in soweit anerkannt, daß er nicht um die Zustimmung seiner Kinder, seiner zukünftigen Erben, fragen müsse. Trotzdem seien die Transaktionen weitgehend ungültig, da das Recht den Kindern einen bestimmten Anteil in ihres Vaters Vermögen garantiere. In ihrer Gesellschaft werde nämlich die Verwandtschaftsbeziehung zwischen Vater und Kindern als so eng betrachtet, daß er ihnen eigentlich nichts, bzw. nur einen sehr 
kleinen Teil seines Vermögens, entziehen dürfe. Um nun einer allzu leichten Umgehung dieser Regel vorzubeugen, sei der Vater schon zu Lebzeiten in seiner Freiheit beschränkt, Schenkungen aus seinem Vermögen zu machen, um so seine Erben, oder einen Teil seiner Erben, um den Genuß seiner Güter zu bringen. Auch seien Schenkungen zwischen Ehegatten nicht erlaubt. In dem Urteil lief es darauf hinaus, daß der Richter berechnete, was der Vater hinterlassen hätte, wenn er keine Schenkungen gemacht hätte. Auch der Laden, den der Vater an seine Tochter und seinen Schwiegersohn verkauft hatte, wurde bei der Berechnung dieses ,eigentlichen“ Nachlasses teilweise berücksichtigt, nachdem bewiesen war, daß der Kaufpreis weit unter dem normalen Wert des Ladens gelegen hatte. Der Unterschied zwischen dem normalen Verkaufswert und dem tatsächlich gezahlten Preis wurde als Schenkung behandelt. Insoweit nun diese Schenkungen die Rechte der klagenden Kinder beeinträchtigten, waren sie ungültig. Das heißt, sie waren nicht automatisch ungültig: Hätten sich die Kinder damit abgefunden, dann wären die Schenkungen wirksam geblieben. Da sie sie jedoch, wie in diesem Fall geschehen, angefochten hatten, mußten sie als von Anfang an ungültig behandelt werden.

Die beiden Urteile zeigen deutlich, daß die individuelle Verfügungsfreiheit in dem Recht der beiden Gesellschaften sehr unterschiedlich geregelt ist. Beide Rechte gestehen dem Individuum zu Lebzeiten eine weitreichende Verfügungsfreiheit zu; es wird nicht die Zustimmung naher Verwandter oder zukünftiger Erben verlangt. In Gesellschaft B können jedoch Verfügungen, die zu Lebzeiten des Erblassers gültig waren, nach seinem Tode wieder ungültig gemacht werden. Der Erblasser ist dadurch schon zu Lebzeiten mehr oder weniger unter Kuratel gestellt, denn das Recht verlangt von ihm, daß er weder durch Testament noch durch Schenkungen seinen Abkömmlingen sein Vermögen entziehe. Diese Regeln zeigen deutlich, wie stark in Gesellschaft B die Beziehung zwischen (Erb)Recht und Verwandtschaft ist. Das Abstammungsprinzip dominiert. Das Individuum ist Teil eines größeren Personenverbandes. Die gegenseitigen Beziehungen sind nicht deutlich verwandtschaftlicher oder vermögensrechtlicher Art und können nicht durch individuelle Verfügungen außer Kraft gesetzt werden. Der rechtliche Schutz der Deszendenten geht selbst so weit, daß noch 20 Jahre zurückliegende und damals vollständiggültige Verfügungen unwirksam gemacht werden können. Kennzeichnend für das Recht von Gesellschaft B ist weiterhin, daß die Unwirksamkeit solcher Verfügungen nicht automatisch eintritt. Ob diese gültig sind oder nicht, hängt letztendlich vom Willen der Deszendenten ab. Das Recht bietet ihnen Schutz. Ob sie jedoch von diesem Schutz Gebrauch machen wollen oder nicht, ist ihre eigene Angelegenheit. Bei dieser rechtlichen Regelung steht deutlich das persönliche Interesse der Verwandten im Vordergrund, nicht das der Gesellschaft als ganzer. Schenkungen an Ehegatten sind prinzipiell ungültig. Hierbei ist zu bedenken, daß dergleiche Schenkungen an eine Person, die nicht zum Abstammungsverband des Schenkers zählt, den Deszendenten des Schenkers ebenfalls einen Teil ihres zu erwartenden Nachlasses zu entziehen drohen.

In Gesellschaft A ist die Rechtslage ganz anders. Hier ist das Individuum unabhängig, nicht durch Verwandtschaftsbeziehungen beschränkt. Seine Verfügungsfreiheit zu Lebzeiten wird konsequent anerkannt, und Verfügungen zum Nachteil zukünftiger Erben sind ohne weiteres gültig. Auch wird den Verwandten nach dem Tod keine Möglichekeit gegeben, dergleiche Verfügungen anzufechten. Das Prinzip, daß das Erbrecht erst nach dem Tod entsteht, ist hier sauber durchgeführt. Anders als in Gesellschaft B ist keine Korrektur in der Form einer zwar erst nacht dem Tode möglichen, jedoch in die Zeit vor dem Tod zurückwirkenden Beschränkung der individuellen Verfügungsfreiheit möglich.

Diese kurze vergleichende Analyse erlaubt eine erste vorläufige Schlußbetrachtung. In dem Bereich der Vermögensrechte und -pflichten, zugespitzt in unserem Beispiel auf die Verfü- 
gungsfreiheit, sehen wir einen deutlichen Unterschied zwischen den beiden Gesellschaften. Die für ,,primitive Gesellschaften“ und das ,,ancient law" "5 so charakteristische undifferenzierte Verschmelzung von persönlichen (personenrechtlichen)und vermögensrechtlichen Beziehungen ist in Gesellschaft A mit ihrer weitgehenden Anerkennung der individuellen Verfügungsfreiheit aufgehoben. In Gesellschaft B, nach deren Recht die Verfügungsfreiheit retroaktiv eingeschränkt werden kann und wo die Deszendenten einen so weitgehenden Schutz genießen, liegt dagegen noch eine starke Verknüpfung von Vermögens- und Verwandschaftsbeziehungen vor. Wenn wir also soziale Differenzierung als ein Kriterium von „,Modernität“ anerkennen, so ist das Recht von Gesellschaft A deutlich ,,moderner" als das von Gesellschaft B.

\section{III}

Lassen Sie mich nun die Anonymität der beiden Gesellschaften lüften. Gesellschaft B mit ihrem traditionellen Recht und der starken Betonung von Verwandtschaftsbeziehungen ist Holland. Gesellschaft A, das Beispiel von ,,modernem“ Recht, sind die Minangkabau in West-Sumatra und ihr heutiges Adat-Gewohnheitsrecht. Der Streitfall, den ich hier wiedergegeben und in einigen, für das Hauptproblem jedoch unwichtigen Einzelheiten verändert habe, wurde 1970 durch ein Indonesisches Gericht in Bukit Tinggi entschieden. Diese Entscheidung wurde später durch das Berufungsgericht in Padang aufrechterhalten ${ }^{6}$. Die Begründung meines Richters $A$ habe ich fast wörtlich aus dem genannten Urteil übernommen und mit Ausführungen aus einem 1972 in einem ähnlichen Fall ergangenen Urteil ergänzt ${ }^{7}$. Die Begründung, die ich meinem Richter B in den Mund gelegt habe, habe ich selbst konstruiert aus dem Text der relevanten Paragraphen des holländischen Bürgerlichen Gesetzbuches und aus Zitaten aus führenden holländischen Lehrbüchern über Erbrecht ${ }^{8}$.

Ich könnte mir denken, daß viele von Ihnen durch das Ergebnis meines Vergleichs überrascht worden sind, auch mag der eine oder andere das westliche Recht - das deutsche Recht z. B. ist nicht grundsätzlich anders ${ }^{9}$ - in der etwas verfremdeten Darstellung erkannt haben. Wie kommt es, daß das holländische Recht so ,,traditionell" und , ,altmodisch“ "ist, obschon man in Holland doch offensichtlich in einer modernen Industriegesellschaft lebt? Und weshalb macht gerade das Minangkabausche Adatrecht, das durch seine Betonung gerade der

5 Vergl. Maine 1905: 230, Goody 1962: 285 ff., Gluckman 1972• 94, Bloch 1975: 204.

6 Fall 49/1970 aus dem Pengadilan Negeri (staatliches Gericht 1. Instanz) Bukit Tinggi und Fall 52/1972 aus dem Pengadilan Tinggi (staatliches Berufungsgericht) Padang.

7 Fall 16/1972 aus dem Pengadilan Negeri Bukit Tinggi. Ich habe diese Fälle und die durch sie aufgeworfenen Probleme ausführlich diskutiert in von Benda-Beckmann 1979: $330 \mathrm{ff}$.

8 Für die ,Urteilsbegründung“ und „Analyse des Rechts von Gesellschaft B“ habe ich mich auf Textstellen aus Asser-Meijers-van der Ploeg 1976: 131, 133, 147, 150, und Pitlo - van der Burght 1977: 86-89, 97, 112-114 gestützt. Die Ansprüche der legitimarissen, der gesetzlich geschützten Erben, sind in den Artikeln 960 ff. des Niedersächsischen Bürgerlichen Gesetzbuch (B. W.) geregelt. Bei einem Kind beträgt die legitieme portie die Hälfte des gesetzlichen Erbteils, bei zwei Kindern 2/3, bei drei oder mehr Kindern 3/4. Art. 967 B. W. legt fest, daß die Einbeziehung von Schenkungen nur auf Verlangen der legitimarissen einuritt. Art. 968 B. W. enthält die Regeln über die Berechnung des zugrunde gelegten Nachlasses. Das Verbot von Schenkungen zwischen Ehegatten ist in Art. 1715 B. W. niedergelegt. Ich bin mir durchaus im klaren, daß ich dieser Regel in meiner ,Analyse“ eine sehr sekundäre Zweckbestimmung unterstellt habe. Primär dient das Schenkungsverbot dem Schutz der Gläubiger von Ehegatten.

9 Nach niederländischem Recht geht der Schutz der Legitimarissen jedoch weiter als der Schutz, den die Pflichtteilsberechtigten nach deutschem Recht genießen ( $\$ 2303$ ff. BGB).

a) Wie in FN 8 bereits erwähnt, kann die legitieme portie 3/4 des gesetzlichen Erbteils betragen, während der Pflichtteilsanspruch nach deutschem Recht in jedem Fall die Hälfte des gesetzlichen Erbteils betrifft, $\$ 2303$ BGB.

b) Nach deutschem Recht erhalten die Pflichtteilsberechtigten lediglich eine schuldrechtliche Forderung gegen die Erben, $\$ 2318$ BGB. Nach niederländischem Recht dagegen gibt die legitieme portie nach herrschender Lehre (Pitlo 1966: 448) einen sachrechtlichen Anspruch auf die zum Nachlaß gerechneten Güter. Art. 972 B. W. legt ausdrücklich fest, daß unbewegliche Sachen in natura zurückgegeben werden müssen.

c) Anders als das deutsche Recht, das mit einem Zeiraum von 10 Jahren vor dem Tod des Erblassers arbeitet (\$2325 BGB), gibt es im niederländischen Recht keine zeitliche Grenze für die Einbeziehung von Schenkungen. 
Abstammungsbeziehungen so bekannt ist, einen so viel ,,moderneren“ Eindruck als das holländische Recht? Aber, als ebenso rhetorische Gegenfrage, weshalb eigentlich diese Überraschung? Sollten wir das Ergebnis eigentlich nicht erwarten? Schließlich geht es um das heutige Minangkabausche Adatrecht und um ein 1838 kodifiziertes und seitdem, zumindest was unser Problem betrifft, nicht wesentlich geändertes Gesetzesrecht. Und schließlich haben wir es in Minangkabau mit einem ungeschriebenen Recht zu tun, das sich relativ leicht an ökonomische und politische Veränderungen anpassen kann, wohingegen die Prozesse von Gesetzesänderungen in westlichen Ländern bekanntermaßen langwierig und mühsam sind. Wäre es deshalb nicht eher zu erwarten, daß das Minangkabausche Adatrecht moderner als das holländische Recht ist?

Es geht mir jedoch nicht darum, zwei sich widersprechende Klischees über westliches und nicht-westliches Recht einander gegenüberzustellen. Ich will auch nicht behaupten, daß das holländische Recht ganz allgemein ,,altmodischer" sei als das Minangkabausche Adatrecht. Das gewählte Beispiel ist auch nur in beschränktem Maße kennzeichnend für die beiden Rechte. Der gegenwärtige Stand des Minangkabauschen Rechts über die Verfügungsfreiheit bezieht sich lediglich auf die Vermögenskategorie der durch eigene Arbeit erworbenen Güter, nicht auf die Erb- und Familiengüter; außerdem ist dieser Zustand erst vor recht kurzer Zeit erreicht worden, und es bleibt abzuwarten, ob diese schrankenlose Verfügungsfreiheit nicht doch noch korrigiert wird. ${ }^{10}$ Und die gegenwärtige Ausgestaltung des Rechtsinstituts der ,legitimen Erbportionen“ im holländischen Recht ist seit längerer Zeit umstritten und wird wahrscheinlich in der Neugestaltung des holländischen Bürgerlichen Gesetzbuches modifiziert werden. ${ }^{11}$

Dennoch bleibt der Vergleich lehrreich. Wenn wir uns über sein Ergebnis wundern, so zeigt das nur, wie sehr wir in Stereotypen denken, wenn wir über ,,traditionelles“ und ,, modernes" Recht sprechen. Dies in zweierlei Hinsicht: Zum ersten geht es um unser Wissen bzw. Nicht-Wissen: Beim genauen Hinsehen zeigen sich sowohl das Recht der traditionellen wie auch der Industriegesellschaft ganz anders als wir es uns eigentlich vorgestellt hatten. Dies führt uns vor Augen, wie wenig sinnvoll es ist, in bezug auf das Recht von ,,modern“ und ,,traditionell“ zu sprechen. ${ }^{12}$ Zum anderen liegen unsern Gedanken ganz bestimmte Vorstellungen über den Zusammenhang von Recht und wirtschaftlicher Entwicklung zugrunde. Kernpunkt dieser Gedanken ist die Assoziation zwischen einem bestimmten Wirtschaftssystem und dem geltenden Recht. Das äußert sich in Úberlegungen wie: ,,Holland hat ein modernes ökonomisches System, also wird es wohl auch ein diesem angemessenes Recht haben. Gesellschaften, die vorwiegend Subsistenzwirtschaft betreiben, haben ein dieser Wirtschaftsform angemessenes Recht. Soll sich nun das Wirtschaftssystem ändern, z. B. im Sinne des ,Entwicklungsgedankens“ von der Subsistenzwirtschaft zur Marktwirtschaft, so muß das Recht dieser neuen Wirtschaftsform angepaßt werden. Oder: verordnet man der Gesellschaft ein an der Marktwirtschaft orientiertes, gleich modernes, gleich westliches Recht, dann wird sich das Wirtschaftssystem schon in diese Richtung entwickeln."

10 Vergl. von Benda-Beckmann 1979: 330 ff. Es fragt sich, ob der Mahkamah Agung, die höchste Indonesische Kassationsinstanz, die zitierten Urteile aufrechterhalten würde. Für andere Gebiete Indonesiens wurde jedenfalls eine so weitgehende Verfügungsfreiheitnicht anerkannt. So wurde in einem Fall aus dem Priangan-Gebiet in West-Java eine Reihe von Schenkungen, die eines der Kinder des Erblassers benachteiligten, schlichtweg für nichtig erklärt, ,,da in Streit mit dem Gerechtigkeitsgefühl und dem dort geltenden Adatrecht “ (Pengadilan Negeri Bandung, Fall 483/1958; bekräftigt durch den Mahkamah Agung, Fall 391 K/Sip/1969. Beide Entscheidungen sind publiziert in Jurisprudensi Indonesia 1970 I: 41-54).

11 So schreiben Pitlo und van der Burght: ,, In Anbetracht der heutigen gesellschaften Entwicklungen scheint es nicht gewagt zu sein zu behaupten, daß die legitieme portie ihre längste Zeit gehabt haben sollte“ (1977: 89).

12 Vergl. auch Bryde: ,Das Leitbild ,Modernität' wurde aus dem, i. d. R. idealisierten, Vorbild der entwickelten Gesellschaften gewonnen, was die Gefahr beinhaltete, daß ganz unreflektiert alle in den Metropolen vorgefundenen Phänomene an dem Prestige der Modernität teilhatten, auch wenn sie als altmodische europäische,Stammesbräuche' richtiger charakterisiert wären. ,Modern' erscheinen dann nicht nur Auto und Eisenbahn, sondern auch die Perücke des englischen Richters (1977: 120). "Vergl. auch Moore $1972: 55$. 
Zugegeben: Ich habe das Stereotyp hier etwas grob formuliert und will es nicht ohne weiteres meinen Zuhörern unterstellen. Doch herrscht dieses Denkmuster bei einem großen Teil derjenigen, in deren Händen die Gestaltung oder Mitgestaltung der Rechtspolitik liegt. Ich will mich auf zwei, wie ich glaube, weitgehend repräsentative Zitate beschränken. Zur Rolle des traditionellen Rechts, Peider Könz, ehemals Vize-Direktor des International Legal Development Center in New York:

„Wo man traditionelles Recht weitergelten ließ, verfügte es weder über hinreichende Durchsetzungsmöglichkeiten noch über Mechanismen, die seine Anpassung an Entwicklungsnotwendigkeiten sicherstellen könnten. Innerhalb der traditionellen Rechtssysteme gab es kaum einen Druck in Richtung Innovation. "13

Und kennzeichnend für die an die Einführung modernen Rechts geknüpften Erwartungen sind die Worte Präsident Bandas von Malawi, der bei der Verabschiedung der nach westlichem Modell orientierten Bodenrechtsreformgesetze sagte:

„Wenn diese Gesetze verabschiedet sind und ausgeführt werden, dann werden sie unsere Landwirtschaft revolutionieren und unser Land von einem armen in ein reiches Land verändern." 14

Die Erfahrung in vielen Ländern der Dritten Welt hat uns gelehrt, daß die aus beiden Zitaten sprechenden Annahmen falsch sind. Die traditionellen Rechte haben sich weitgehend mühelos neuen sozialen und wirtschaftlichen Formen anpassen können; auf das Minangkabausche Beispiel werde ich später noch zurückkommen. Die Einführung des sogenannten modernen westlichen Rechts hat jedoch fast nirgends die damit verknüpften Erwartungen erfüllt. ${ }^{15}$ Woran liegt das nun? Ich meine, es liegt zumindest weitgehend daran, daß man sich über die Eigenschaft von Recht und über den Zusammenhang von Recht/Rechtsentwicklung und sozio-ökonomischer Entwicklung nicht klar genug ist.

Allem Recht - wie man es auch im einzelnen definieren möge - ist eigen, daß es die Autonomie der Gesellschaftsmitglieder in einem gewissen Grade anerkennt und beschränkt. ${ }^{16}$ Die „Anerkennung“" kommt meist in der Form von Rechten, Berechtigungen zum Ausdruck; die Beschränkungen in den Berechtigungen der anderen Gesellschaftsmitglieder und in den Befugnissen der Gesamtgesellschaft, so wie diese durch die gesellschaftlichen politischen Institutionen verwaltet werden. Aber was auch immer der Inhalt von Rechtsnormen ist, sie sind stets relativ in ihrer Anerkennung und Beschränkung; immer bleibt ein Freiraum, in dem die Gesellschaftsmitglieder tun und lassen können, was sie wollen und können; d. h. frei von rechtlichen Beschränkungen. Was sie innerhalb dieses Freiraums tun, ist im wesentlichen von anderen Faktoren und Einflüssen abhängig; von moralischen, religiösen, politischen und kulturellen Faktoren. Ändern sich nun diese Faktoren, so wird das in der Regel auch die Handlungsweise der Gesellschaftsmitglieder ändern.

Wenn der durch das Recht zugelassene Handlungsfreiraum einigermaßen weit ist, so ist deutlich:

1. daß sich ganz erhebliche Veränderungen in den Bereichen des sozialen und wirtschaftlichen Handelns vollziehen können, ohne daß das Recht sich ,,anpassen“ oder gar ,ändern" müßte, und

13 Könz 1969: 94. Für die kritische Analysen derartiger Behauptungen siehe auch Burg 1977: 502, Bryde $1976: 108$ ff.

14 Malawi Hansard: 4th Session, 4th Meeting, April 1967, p. 402.

15 Bryde 1977: 126 ff. mit Nachweisen.

16 Ich habe mich an anderer Stelle bemüht, auf dieser Basis ein für die anthropologische (vergleichende) Rechtsforschung taugliches Rechtskonzept zu entwickeln, s. von Benda-Beckmann 1979: $25 \mathrm{ff}$. 
2. daß es bei einer gewünschten Veränderung im sozialen und wirtschaftlichen Bereich nicht in erster Linie auf das Recht bzw. eine Rechtsänderung ankommt, sondern primär auf entsprechende soziale und wirtschaftliche Stimuli.

A uf unser Beispiel der Verfügungsfreiheit bezogen: Die Tatsache, daßein Rechtssystem einen bestimmten Grad von Verfügungsfreiheit zuläßt, sagt noch gar nichts darüber aus, ob und wie von dieser Freiheit Gebrauch gemacht wird.

Lassen Sie mich von diesen recht abstrakten Gedanken wieder zu konkreten Beispielen kommen. Eine der besten Analysen, die auf den gerade genannten Grundeinsichten basiert, stammt von dem österreichischen Rechtssoziologen Karl Renner. In seinem Buch „Die Institute des Privatrechts und ihre soziale Funktion“ (1929) beschreibt und analysiert Renner die Beziehung zwischen Rechtswandel und wirtschaftlicher Entwicklung in Europa, insbesondere in der Periode vom ausgehenden Spätmittelalter bis zum Ende des 19. Jahrhunderts, oder spezifischer: die Periode, in der sich der Wandel von der einfachen Warenproduktion zur kapitalistischen Produktionsweise vollzog.

Renner beschreibt zunächst den Mikrokosmos der einfachen Warenproduktion und dessen Basis, das ,,Erb und Eigen“; wo Produktionsort, Distributionsort und Konsumptionsort in der Regel zusammenfallen, wo der Produzent Eigentümer seiner Produktionsmittel und seiner Produkte ist und seine Arbeit selbst kontrolliert. Mit Renners Worten:

„,Die sachlichen Voraussetzungen des gesamten Produktions- und Reproduktionsprozesses sind zusammengestellt zu einer organischen Einheit von Dingen, welche Welt durch die Individualität des Eigners ihre Individuation erhält. Der Kosmos ist durch seine wirtschaftliche Zweckbestimmung, nicht durch rechtliche Satzung, zur Einheit geworden" ${ }^{17}$

Diese wirtschaftliche Zweckbestimmungund die sie ausmachenden Produktions- und Reproduktionsprozesse nennt Renner in mehr allgemeiner Weise die ,,ökonomische Funktion des Rechtsinstituts“, oder das ,,Substrat der Norm“. Bei der Beschreibung des Wandels des Wirtschaftssystems macht Renner nun deutlich, daß dieser Wandel im wesentlichen ein Wandel der ökonomischen Funktion des Eigentumsrechts ist, sich die das Institut des Eigentums ausmachenden Rechtsnormen dagegen kaum geändert haben. Diese Art des Wandels nennt Renner dann - ganz allgemein - den Funktionswandel des Rechts: die Erscheinung, daß sich auf Grund außerrechtlicher, z. B. sozialer und wirtschaftlicher Faktoren die sozialen und wirtschaftlichen Prozesse innerhalb eines Teilrechtssystems ändern, ohne daß sich die Normen dieses Teilrechtssystems ändern. Der Funktionswandel wird deutlich vom Normwandel abgegrenzt. Normwandel liegt vor, wenn sich die Rechtsnormen selbst auf Grund sozialer, wirtschaftlicher und politischer Prozesse ändern.18

\footnotetext{
17 Renner 1929: 32.

18 Renner 1929: 5 ff. Bryde weist darauf hin, daß Weber und Parsons die westeuropäische Rechtstradition für eine entscheidende Bedingung der Entstehung von Kapitalismus und Industriegesellschaft in Westcuropa gehalten haben (1977: 121). Es ist Renners Verdienst, mit seiner Arbeit mehr Klarheit in den Zusammenhang zwischen dem Recht und der wirtschaftlichen Entwicklung gebracht zu haben. Der Ubergang zur kapitalistischen Wirtschaft war weitgehend innerhalb des vor-kapitalistischen Rechts möglich. Der Kapitalismus selbst bzw. die ihn tragenden Kräftegestaltet dann das Recht nach seinen Erfordernissen und seiner Logik gemäß weiter um. Vergl. auch Balbus 1978.
} 
Ich meine, daß die Unterscheidung zwischen Normwandel und Funktionswandel ${ }^{19}$ von ganz entscheidender Bedeutung für die Analyse der Prozesse der „,Rechtsentwicklung“ ist und uns auch bei der Analyse des Zusammenhangs zwischen sozio-ökonomischer und Rechtsentwicklung in traditionellen Gesellschaften gute Dienste leistet. Kommen wir zurück auf das Minangkabausche Beispiel. In Minangkabau haben sich in den letzten $150 \mathrm{Jah}$ ren große Veränderungen vollzogen, die ich ganz kurz skizzieren will. ${ }^{20}$

Im traditionellen Minangkabauschen Adatsystem wurden im wesentlichen zwei Vermögenskategorien unterschieden. Die sozial und ökonomisch wichtigsten Güter - es geht hier vor allem um Reisland - waren die pusako-Güter, das Familien- und Erbgut der matrilinearen Abstammungsgruppen. Gebrauchsrechte an pusako wurden nach bestimmten Regeln unter den Gruppenmitgliedern verteilt und innerhalb der Gruppe vererbt. Pusako-Land durfte nicht permanent verfremdet werden. Zugestanden waren lediglich zeitlich begrenzte Verpfändungen, wozu alle Gruppenmitglieder ihre Zustimmung geben mußten.

Die andere Güterkategorie waren die pancaharian-Güter, Vermögen, das Individuen zu Lebzeiten durch eigene Arbeit selbst erworben hatten. Nach dem Adat wurden diese Güter nach dem Tod des Erwerbers zu pusako, zu Erbgütern der matrilinearen Abstammungsgruppe. Die Verfügungsfreiheit des Erwerbers über diese Güter war recht groß. Einschränkungen gab es jedoch hinsichtlich Schenkungen. Da diese der Abstammungsgruppe zukünftiges pusako zu entziehen drohten, waren Schenkungen - es ging hier fast immer um Schenkungen an die Kinder des Erwerbers - an die Zustimmung der Gruppenmitglieder gebunden.

Dies ist in wenigen Worten die Ausgangslage. Mit der Unterwerfung unter die holländische Kolonialherrschaft wurden die Minangkabau in zunehmendem Maß neuen Einflüssen ausgesetzt. Ein koloniales Verwaltungssystem wurde eingeführt und die politische und soziale Macht der Oberhäupter der Abstammungsgruppen eingeschränkt. Die Dorfökonomie wurde immer weiter an das internationale Wirtschaftssystem angeschlossen. In der ersten Phase der kolonialen Wirtschaftspolitik wurden die Bauern gezwungen, Kaffee zu produzieren, den die Holländer auf dem europäischen und Weltmarkt verkauften. Der Kaffeeanbau ging, anders als der Reisanbau, in kleineren Produktionseinheiten vor sich; er war hauptsächlich eine Sache der Männer. Das durch den Kaffeeanbau verdiente Geld war im wesentlichen unabhängig vom pusako erworben, es war pancaharian, selbst erworbenes Gut. Gegen Anfang dieses Jahrhunderts änderte sich die koloniale Wirtschaftspolitik. Der Zwangsanbau von cash-crops wurde aufgegeben, dafür die Schatzkiste mit einer neu eingeführten Grundsteuer gefüllt. Gleichzeitig wurde der Import von ausländischen Waren verstärkt zugelassen. Hierdurch wurden neue Bedürfnisse nach Geld geschaffen; zugleich wurden durch die Ausweitung des kolonialen Verwaltungs- und Wirtschaftssystems neue Möglichkeiten geboten, Geld zu verdienen. Das selbst-erworbene Gut, die pancaharian-Güter, bekamen so eine viel größere wirtschaftliche Bedeutung als sie vorher gehabt hatten. Dieser Einfluß konnte durch das pusako-System insoweit aufgefangen werden, daß das neue pancaharian zu einer ständigen Vermehrung des pusako-Komplexes führte; pancaharian-Güter wurden nach dem Tode des Erwerbers ja zu pusako. Im Laufe der Entwicklung wurde dieser Prozeß

19 Im folgenden behalte ich den Rennerschen Begriff des ,Funktionswandels“ bei, obschon er, wie hier betont werdensollte, erheblich von den in der Soziologie und Anthropologie gebräuchlichen Funktionsbegriffen abweicht.

20 Ich habe diese Entwicklungen ausführlich beschrieben in von Benda-Beckmann 1979, vor allem auf S. $274 \mathrm{ff}$. und S. $326 \mathrm{ff}$. Vergl. hierzu auch: Evers 1975, Guyt 1936, Kahn 1975, Maretin 1961, Naim (ed.) 1968, Prins 1953, 1954, Sarolea 1920, Schrieke 1955, Tanner 1971, van Vollenhoven I: 246 ff., Willinck 1909. 
jedoch weitgehend in sein Gegenteil verkehrt: Anstatt daß dem pusako-Komplex neue Vermögenswerte zugeführt wurden, wurden die Rechte, die Gebrauchsrechte am pusako-Land, in zunehmendem Maße in pancaharian-Rechte umgesetzt. Die Gebrauchsrechte am Reisland wurden dadurch immer leichter übertragbar. Die traditionellen gruppeninternen Verteilungsmechanismen wurden so langsam von übertragbaren Gebrauchsrechten mit pancaharian-Charakter überlagert. Auch im sozialen Leben vollzogen sich Änderungen. Immer seltener wohnten, wie früher üblich, ganze matrilineare Abstammungsgruppen in den traditionellen Familienhäusern zusammen; vermehrt wurden kleinere Häuser gebaut, in denen Kleinfamilien wohnten. Die neuen Wohnverhältnisse und die zunehmend auf pancaharian-Rechten beruhenden Produktionsverhältnisse ließen das Band zwischen Ehegatten und ihren Kindern stets enger werden. Männer gingen immer häufiger dazu über, große Teile ihres pancaharian-Vermögens ihren Kindern zu schenken.

Die veränderten sozio-ökonomischen Verhältnisse führten zu einer Änderung im Minangkabauschen Adatrecht. Die wesentliche Änderung lag in einer immer weitergehenden rechtlichen Anerkennung der individuellen Verfügungsfreiheit über pancaharian-Güter, im wesentlichen, wie gesagt, von Schenkungen an die Kinder des Erwerbers. Oder, komplementär ausgedrückt, in einer fortschreitenden Beschränkung des Rechts der anderen Mitglieder der Abstammungsgruppen, durch ihre Nicht-Zustimmung zu der beabsichtigten Verfügung eine rechtlich wirksame Verfügung zu verhindern.

Dieser Prozeß wurde durch die holländischen Kolonialgerichte unterstützt, die hierin den ,,unvermeidlichen“ UUbergang von dem , unnatürlichen“ Mutterrechtssystem zum ,,natürlichen“ und ,,modernen“ bilateralen Erbrechtssystem sahen. Um diesen wünschenswerten Prozeß zu beschleunigen, mußten die Einspruchsrechte der Gruppenmitglieder abgeschafft werden. Hiermit wurde der Grundstein für die in meiner Fallgeschichte beschriebene schrankenlose Verfügungsfreiheit gelegt. Den Holländern, und auch den Minangkabau selbst, ging es zwar primär darum, eine bestimmte Gruppe von Berechtigten, die matrilinearen Verwandten, durch eine neue, die Kinder, zu ersetzen. Der Weg dahin führte über die Aberkennung der Einspruchsrechte der ursprünglich Berechtigten, ohne daß jedoch zugunsten der neuen Berechtigten vergleichbare Einspruchsrechte eingeführt wurden. ${ }^{21}$ Mit der Anerkennung der Verfügungsfreiheit über pancaharian-Güter war die Basis für einen weiteren, erst kürzlich eingetretenen Normwandel gelegt: Die Anerkennung von Erbrechten der Kinder an pancaharian-Gütern; also auch in den Fällen, in denen die Erwerber ihr pancaharian nicht schon zu Lebzeiten ihren Kindern geschenkt hatten. ${ }^{22}$

Dieser Normwandel ist sicherlich ein wichtiges Element der sozialen und wirtschaftlichen Entwicklung in Minangkabau, durch diese bedingt und sie gleichzeitig beeinflussend. Seine Folgen liegen jedoch primär im Erbrecht. Die bedeutende wirtschaftliche Entwicklung jedoch, die zunehmende Individualisierung und Kapitalisierung des dem Unterhalt der matrilinearen Gruppen dienenden pusako-Komplexes waren weitgehend ohne Änderungen des Minangkabauschen Adatrechts möglich.

Ich will das kurz erläutern. Wie bereits erwähnt, war die befristete Verfremdung von pusako-Gütern in der Form von Verpfändungen weitgehenden Beschränkungen unterworfen. Verpfändungen waren nur in bestimmten Situationen zugelassen, in denen die Abstammungsgruppe als ganze für Gruppenzwecke Geld nötig hatte. Das pusako-Land wurde dann

21 Diese Entwicklung wurde 1930 in dem berühmten Fall des Nachlasses von Dr. Muchtar durch den Hooggerechtshof, das Oberste Gericht der Kolonie, sanktioniert (publiziert in Indisch Tijdschriftvoor het Recht 131: 82 ff., vgl. auch von Benda-Beckmann 1979: 330, 336).

22 Diese neue Rechtsregel wurde 1968 durch den Mahkamah Agung expliziet als das ,,neue Adatrecht“ von Minangkabau niedergelegt (Jurisprudensi Indonesia 1969 III: 17-33; vgl. von Benda-Beckmann 1979: 337-340). Uber die Gestaltung des Erbrechts anderer Indonesischer Gesellschaften durch den Mahkamah Agung s. Lev 1962. 
durch die Gruppe, vertreten durch ihr Oberhaupt, verpfändet. Im Prinzip wurde das Land später durch die ganze Gruppe wieder eingelöst und danach in Einklang mit den traditionellen Verteilungsmechanismen wieder unter die Gruppenmitglieder verteilt. Nach Ablauf einer Minimumfrist hatte der Pfandnehmer jedoch das Recht, das Land zurückzugeben und die Einlösung zu verlangen. Verfügte nun die Gruppe nicht über ausreichende Mittel, so konnte der Pfandnehmer das Land an einen Dritten weiterübertragen. Eine solche Weiterübertragung war ohne die Zustimmung des ursprünglichen Pfandgebers nicht möglich - aber diese Zustimmung konnte nicht verweigert werden, wenn das Land zur Einlösung angeboten worden war. Úberdies behielt die pfandgebende Gruppe auch gegenüber der dritten Partei das Recht, jederzeit das Land von ihr einzulösen. Auf diese Weise war also eine Úbertragung von Gebrauchsrechten an pusako-Land möglich, ohne daß die strikten Beschränkungen des Rechts eingriffen. Eine weitere Situation, in der dies möglich war, war gegeben, wenn die pfandgebende Gruppe nicht über ausreichende Mittel verfügte, um das Pfand einzulösen, wohl aber einzelne Gruppenmitglieder. Wollte oder konnte die Gruppe das Land nicht einlösen, so konnte es dem einzelnen Gruppenmitglied nicht verwehrt werden, das Land für sich selbst einzulösen. Das Land behielt auch in diesem Falle seinen pusako-Charakter, und die übrigen Gruppenmitglieder hatten auch das Recht, sich durch die nachträgliche Zahlung eines Anteils der Einlösesumme einen Anteil an dem Land bzw. den Gebrauchsrechten daran zu sichern. Aber solange dies nicht geschah, war das durch die Einlösung erlangte Gebrauchsrecht an dem pusako-Land exklusiv für denjenigen, der es eingelöst hatte.

Dies sind Regeln des traditionellen Minangkabauschen Vermögensrechts, die sich bis heute nicht geändert haben. In der ,, wirtschaftlichen Funktion“ dieser Regeln ist jedoch eine große Veränderung eingetreten. Früher waren es Gruppen, die pusako verpfändeten und das verpfändete Gut wieder einlösten; meist mit Mitteln, die pusako-Charakter hatten, wie Geld, das durch Ưberschuß des pusako-Reislandes verdient war. Heute sind es hauptsächlich Individuen, die, ganz in Einklang mit den obengenannten Regeln, pancaharian-Geld in Einlösungen und Weiterübertragungen von verpfändetem pusako-Land investieren und über die dadurch erworbenen Gebrauchsrechte ebensof rei verfügen können wie über andere pancaharian-Güter. Die Folge hiervon ist, daß die Gebrauchsrechte an pusako-Reisland sehr mobil geworden sind und daß die traditionellen Verteilungsmechanismen und die mit ihnen verbundenen Produktionsverhältnisse drastisch an Bedeutung verloren haben und durch Gebrauchsrechte überlagert werden, deren primäre Legitimation das in sie investierte Geld ist. $^{23}$

Soweit die kurze Analyse des Norm- und Funktionswandels in Minangkabau. Ich hoffe, daß sie deutlich gemacht hat, wie wichtig diese Unterscheidung für das Verständnis der gesamtgesellschaftlichen Entwicklung ist und wie wichtig sie vor allem dann ist, wenn man sich Gedanken darüber macht, ob und inwieweit neues Recht zur Erreichung betimmter sozio-ökonomischer Verhältnisse erforderlich ist. Die mir zur Verfügung stehende Zeit erlaubt es mir nicht, näher darauf einzugehen, inwieweit das Minangkabausche Beispiel repräsentativ für Entwicklungsprozesse in anderen Gesellschaften in Ländern der Dritten Welt ist. Ich nehme an, daß das weitgehend der Fall ist; diese Erwartung müßte jedoch durch

23 Uber die Veränderungen der Produktions- und Reproduktionsverhältnisse in afrikanischen Gesellschaften als Folge des eindringenden Kapitalismus und der dadurch bewirkten Vergeldlichung der Brautpreissysteme s. Bohannan 1967, Rey 1971. 
neue Untersuchungen und Analysen bestätigt werden - Analysen, die auf das in meinem Vortrag genannte Vorverständnis von Recht und Entwicklung basiert sein müßten. Solche Untersuchungen sind jedoch bislang noch recht selten, weil - und hiermit komme ich zum letzten Punkt meines Vortrags - das Vorverständnis, das sie voraussetzen, ganz allgemein nicht zum theoretischen und methodologischen Rüstzeug der Anthropologen gehört; oder etwas vorsichtiger gesagt, zumindest soweit sich Anthropologen mit Recht befassen.

Es sollte deutlich geworden sein, daß die Kategorie Recht, die ich in meinem Vortrag verwendet habe, von den sozialen Prozessen getrennt werden muß, die es schaffen, manipulieren, verändern, und die man im Rennerschen Sinne seinen Substrat, seiner sozialen Funktion zuordnen müßte. Erst diese Trennung macht die Annahme einer dialektischen Beziehung zwischen dem Recht und den genannten sozialen Prozessen möglich. Die in der (Rechts-)Soziologie und Anthropologie vorherrschenden Definitionen des Begriffs Recht beruhen nun aber gerade auf einer Verknüpfung von abstrakten Regeln und sozialen Prozessen. In dem diesen Definitionen zugrunde liegenden, meist evolutionistisch orientierten Denkmodell wird das Recht als ein Ordnungstypus charakterisiert, das sich von anderen Ordnungstypen wie Brauch, Sitte oder custom durch eine bestimmte Sanktionsart bei Normabweichungen unterscheidet. Gemeinsam ist jedoch allen Ordnungstypen, daß sie auf Verhaltensregelmäßigkeiten, statistischen Normen, behaviour patterns beruhen. Auf Grund bestimmter sozialpsychologischer Prozesse entwickeln diese Verhaltensregelmäßigkeiten einen Soll-Charakter; sie werden zu sozialen Normen. Ihren spezifisch rechtlichen Charakter erhalten sie dann durch die besondere Art der Sanktion in den Fällen, in denen die Gesellschaftsmitglieder von der Norm abweichen. ${ }^{24}$

Dies ist, in wenigen Worten, das Paradigma, das der rechtssoziologischen und -anthropologischen Normtypologie zu Grunde liegt. Wenn auch der spezifisch rechtliche Charakter der Norm in der Sanktionierung liegt, so erhält sie ihren eigentlichen Inhalt jedoch als soziale Norm im Sinne einer vorgeschriebenen Verhaltensregelmäßigkeit. Änderungen in den Verhaltensregelmäßigkeiten müssen also nach diesem Modell zu einer Änderung der Rechtsnorm führen, wenn sie nur in der rechten Form sanktioniert werden. Wir sehen, daß eine saubere Trennung zwischen dem Recht und seinem Substrat hier nicht möglich ist. Das aus diesen Definitionen sprechende Vorverständnis von Recht hat sicher dazu beigetragen, daß Anthropologen sich nicht eingehend mit der Problematik der Rechtsentwicklung in den von ihnen untersuchten Gesellschaften in den Ländern der Dritten Welt befaßt haben.

Und hier liegt, am Ende meines Vortrags, auch die Moral der Geschichte: Die Entwicklung der traditionellen Gesellschaften in den Ländern der Dritten Welt ist nicht nur wissenschaftlich interessant, sondern wird zu Recht als ein brennendes politisch-moralisches Problem empfunden. Die Frage, wie sich das Recht entwickelt und wie Recht im Sinne einer erwünschten Entwicklung eingesetzt werden kann, ist Teil dieses allgemeineren Problems. Doch ich glaube und ich hoffe mit meinem Vortrag deutlich gemacht zu haben, daß wir, bevor wir konkrete Maßnahmen vorschlagen oder durchführen, erst Klarheit über die tatsächlichen Zustände in den betreffenden Gesellschaften gewinnen müssen, d. h. forschen müssen. Und bevor wir das tun, bzw. um das in sinnvoller Weise tun zu können, müssen wir ein theoretisches Repertoire erarbeiten, das uns hilft, die konkreten Zustände zu begreifen, und es uns ermöglicht, Aussagen über Entwicklungszusammenhänge zu machen.

24 Besonders deutlich ist diese Ableitung bei Hoebel. Hoebel beginnt mit behavior patterns und habits, Normen im statistischen Sinne (1954: 11, 14). Diese neutralen statistischen Normen ,take on the quality of the normative“, sie werden zu social norms (1954: 15). Diese sozialen Normen haben rechtlichen Charakter, wenn sie in einer bestimmten Form sanktioniert werden, nämlich durch ,, an individual or group possessing the socially recognized privilege" (Hoebel 1954: 28). Vergl. auch die sehr ähnliche Entwicklung des Rechtstypus bei Geiger, dem die Rechtssoziologie die wohl ausgefeilteste Normtypologie verdankt (Geiger 1964: 49, 62 ff.). 


\section{Literatur}

Allot, A. N.

1962 Judicial and Legal Systems in Africa, London.

Apter, D. E.

1965 The Politics of Modernization, Chicago: University of Chicago Press.

Asser, C., Meijers, E. M. en P. W. van der Ploeg

1976 Erfrecht, Zwolle: Tjeenk Willink.

Balbus, I.

1978 „Commodity Form and Legal Form: An Essay on the Relative Autonomy of Law", in: Reasons, C. E. and R. M. Rich (eds.), The Sociology of Law: A Conflict Perspective, Toronto: Butterworth, pp. 73-90.

Benda-Beckmann, F. von

1970 Rechtspluralismus in Malawi; Geschichtliche Entwicklung und heutige Problematik des pluralistischen Rechtssystems eines ehemals britischen Kolonialgebiets, Ifo-Institut für Wirtschaftsforschung, Afrika-Studien No. 56, München: Weltforum-Verlag.

1979 Property in Social Continuity: Continuity and Change in the Maintenance of Property Relationships Through Time in Minangkabau, West Sumatra, Verhandelingen van het Koninklijk Instituut voor Taal-, Land- en Volkenkunde No. Bloch, M. 86, The Hague: M. Nijhoff.

1975 „Property and the End of Affinity“, in M. Bloch(ed.), Marxist Analyses and Social Anthropology, London: Malaby Press.

Bohannan, P.

1967 ,,The Impact of Money on an African Subsistence Economy“, in G. Dalton(ed.), Tribal and Peasant Societies: Readings in Economic Anthropology, New York, The Natural History Press.

BPHN

1974 Badan Pembinaan Hukum Nasional, Proceedings of the Third National Law Conference held in 1974, Jakarta.

1975 Badan Pembinaan Hukum Nasional, Conclusions of the Seminar on Adat Law and Development, Jakarta.

Bryde, B. O.

1976 The Politics and Sociology of African Legal Development, Frankfurt, A. Metzner.

1977 „Rezeption europäischen Rechts und autozentrierte Rechtsentwicklung in Afrika“, in Afrika Spektrum, Heft 2, pp. 117-130.

Burg, E. M.

1977 „Law and Development: A Review of the Literature \& a Critique of ,Scholars in Self-Estrangement"“, in AJCL 25: 492-530.

Elias, T. O.

1962 Britisch Colonial Law, London.

Evers, H. D.

1975 „Changing Patterns of Minangkabau Urban Landownership“, in BKI 131: 86-110.

Geiger, Th.

1964 Vorstudien zu einer Soziologie des Rechts, Neuwied: Luchterhand. 
Gluckmann, M.

1972 The Ideas in Barotse Jurisprudence, 2nd ed., Manchester: Manchester University Press.

Goody, J.

1962 Death, Property and the Ancestors, Stanford: Stanford University Press.

Guyt, $\mathrm{H}$.

1936 Grondverpanding in Minangkabau, Leiden.

Hoebel, E. A.

1954 The Law of Primitive Man, Cambridge: Harvard University Press.

Hooker, M. B.

1975 Legal Pluralism - An Introduction to Colonial and Neo-Colonial Laws, Oxford: Clarendon Press.

Kahn, J.

1975 „Economic Scale and the Cycle of Petty Commodity Production in West Sumatra", in M. Bloch (ed.), Marxist Analyses and Social Anthropology, London:

Könz, P. Malaby Press, pp. 137-160.

1969 Legal Development in Developing Countries, Proceedings of the American Society of International Law, pp. 91-100.

Lev, D. S.

1962 „The Supreme Court and Adat Inheritance Law in Indonesia“, in AJCL 11: 205-224.

Maine, H. S.

1905 Ancient Law, 10th ed., London: Murray.

Malawi Hansard, The,

Official Verbatim Report of the Debates of Parliament, Zomba: Government Printer.

Maretin, J. V.

1961 „Disappearance of Matriclan Survivals in Minangkabau Family and Marriage Relations", in BKI 117: 168-195.

Merryman, J. H.

1977 Comparative Law and Social Change: On the Origins, Style, Decline \& Revival of the Law and Development Movement", in: AJCL 25: 457-491.

Moore, S. F.

1972 „Legal Liability and Evolutionary Interpretation: Some Aspects of Strict Liability, Self-Help and Collective Responsibility“, in M. Gluckman(ed.), The Allocation of Responsibility, Manchester: Manchester University Press.

Naim, M. (ed.)

1968 Menggali Hukum Tanah dan Hukum Waris Minangkabau, Padang: Center for Minangkabau Studies.

Pitlo, A.

1966 Het Systeem van het Nederlandse Privaatrecht, Derde, Herziene Druk, Haarlem: Tjeenk Willink en Zoon N. V.

Pitlo, A. en G. van der Burght

1977 Erfrecht, Arnhem: Gouda Quint B. V. 
Prins, J.

1953 „Rondom de oude strijdvraag van Minangkabau“, Indonesië 7: 320-329.

1954 Adat en Islamietische Pflichtenleer in Indonesië, Derde Druk, 's-Gravenhage: van Hoeve.

Renner, K.

1929 Die Rechtsinstitute des Privatrechts und ihre soziale Funktion, Tübingen.

Rey, P. P.

1971 Colonialisme, Néo-Colonialisme et Transition a u Capitalisme: Exemple de la ,Comilog' au Congo-Brazzaville, Paris: Maspero.

Sarolea, W. H. A.

1920 „Minangkabausch Adatrecht: De rechtsbevoegdheid van den familietak, zijn goederen en zijn hoofd“, ITR 112: 120-131.

Schrieke, B.

1955 „,The Causes and Effects of Communism on the West Coast of Sumatra“, in: Indonesian Sociological Studies; Selected Writings of B. Schrieke, part 1, The HaTanner, N. gue: van Hoeve.

1971 Minangkabau Disputes, unpublished $\mathrm{PhD}$ Thesis, University of California, Berkeley.

Vollenhoven, C. van

1918-

1933 Het Adatrecht van Nederlandsch-Indië, 3 Delen, Leiden, Brill.

Willinck, G. D.

1909 Het Rechtsleven bij de Minangkabausche Maleiërs, Leiden, Brill. 


\section{Modern law and traditional societies}

BY FRANZ VON BENDA-BECKMANN

The analysis of the relationship between legal and socio-economic change requires a conceptual apparatus in which the concept of law is dissociated from the social processes to which its rules relate. The changes in the property and inheritance law of the Minangkabau of West Sumatra illustrate that important socio-economic changes may occur within the frame work of basically unchanged law. As is shown by a comparison between Minangkabau and Dutch law, the labels "modern" and "traditional/non modern" are highly misleading when applied to the unwritten law of non western societies and its development.

\section{The Role of the Judiciary in Modernizing Law in Africa By HeINRICH SCHOLleR}

The starts by describing the development of modern courts and modern law in Ethiopia which is characterized by a lack of colonial influence in this country. It shows the importance of mixed court jurisdiction in the early stage of modernizing law. This system had to resolve two mayor problems which involved the question of maintaining sovereignty: first, the jurisdiction over foreigners, second, the execution of foreign judgments in an African country. The problem of overcoming the concept of the so-called "duality of law" is also one of the main points of interest. The article stresses that the duality or plurality of law is not due to mere colonial policy, but rather to the process of modernizing law. The process of modernization started always out of a socially marginal position. This is also true for the revolutionary law although the role of courts and law during the Ethiopian revolution is only briefly mentioned.

According to this article the meaning of the term "modernizing law" is not restricted to the law making process itself but extended to the role of law as a part of social engineering. The independence of the law courts is considered an important element in the fulfilment of this function. The function of social engineering includes the task of education, assimilation and emancipation of men and women by modernized law. The task of political education can only be accomplished by law courts if they recognize their creative function and, for that purpose, claim a certain discretion in finding just and equitable solutions.

\section{“Autocentric" development in Ethno - Sociological perspective BY INGEBORG Y. WENDT}

Industrialization in Europe was pursued parallel with a growing philosophical inclination towards rationalism and materialism, i.e. economic development was in accordance with the general mental tendency. - There was wide-spread illiteracy even in England during the time of the "Industrial Revolution", and also rapid growth of population. These historical facts are ignored by theories on development, and wrongly literacy and family planning are considered as preconditions of economic growth in today's developing countries. - Japan, the only successful non-European country, was in seclusion when the rest of the non-European world was under colonial rule. Thus, it preserved its social system including a poor, but sustaining agriculture and its moral values, and retained its material substance. Japan was a well functioning, dynamic entity when it was forced to enter the international scene in $1853 / 54$. In contrast India, representing the former colonies and present developing coun- 